\title{
ORIENTED THIN FILMS FOR NONLINEAR OPTICS
}

\author{
F. KaJZar, A. LoRin \\ Commissariat à l'Energie Atomique, Direction des Technologies Avancées \\ LETI, DEIN/SPE/GCO, CE Saclay, 91191 Gif Sur Yvette Cedex, France
}

\section{J. Le MoIgne}

Instutit de Physique et de Science des Materiaux 23, rue du Loess, 67037 Strasbourg, France

\section{AND J. SzPUNAR}

Department of Mining and Electrical Engineering, Mc Gill University 3450 University Street, Montréal PQ, Canada H3A 2 A7

Nonlinear optical dichroism technique is shown to be an useful tool for the $1 \mathrm{D}$ conjugated polymer chain orientation study. When done as a function of rotation angle it gives the order parameters. These parameters can be also obtained by coupling this technique with linear dichroism method. The technique is illustrated by third harmonic generation on epitaxied polydiacetylene thin films either on single-crystalline or on preoriented ultrathin films by rubbing. Whereas an almost perfect orientation is obtained in the case of epitaxy of p-DCH, poly [1,6-bis $(N$-carbazolyl)-2,4-hexadiyne], on $\mathrm{KAP}$, potassium acid phthalate, single crystal, this is smaller for epitaxy on preoriented substrate. The results are correlated with thin film texture measurements with X-rays. A good overall agreement is seen except that X-ray data show better order than nonlinear optical dichroism. A possible origin of this difference is shortly discussed.

PACS numbers: 42.65.-k, 78.20.-e, 83.80.Es

\section{Introduction}

In recent years one observes an increasing interest in synthesis and nonlinear optical properties characterization of organic molecules in view of their application in electrooptical or all optical devices. It is almost possible now, through molecular engineering, to obtain molecules with defined physico-chemical properties [1] . 
For practical applications the quantity of interest is the macroscopic polarization of nonlinear medium under the external forcing fields. This polarizability can be developed, in dipolar approximation, in the external field power series giving

$$
\boldsymbol{P}=\boldsymbol{P}_{0}+\chi^{(1)}: E+\chi^{(2)}: E E+\chi^{(3)}: E E E+\ldots
$$

where the expansion coefficients are $(n+1)$ rank three-dimensional tensors describing different nonlinear optical (NLO) processes and $\boldsymbol{P}_{\mathbf{0}}$ is the medium permanent polarization. Similarly, on the molecule level, its polarization can be also developed in the forcing field $E$ power series giving

$$
p=p_{0}+\alpha: E+\beta: E E+\gamma: E E E+\ldots
$$

where here again the expansion coefficients $\alpha, \beta, \gamma, \ldots$ are $(n+1)$ rank three-dimensional tensors, with $n$ defined by the electric field $E$ power describing various nonlinear optical responses of the molecule experiencing the electric field $E$ which generally is different from the applied external field due to the screening. $p_{0}$ is the molecule permanent polarization. For centrosymmetric molecules and centrosymmetric structures and within the mentioned dipolar approximation all odd rank tensors are equal to zero. It means that even if the molecule is noncentrosymmetric the odd rank tensor of a bulk medium composed of this molecule may be equal to zero. It shows how important is order in determining the macroscopic nonlinear optical response, which is important for practical applications, as already mentioned. For a single crystal there exists a direct link between macroscopic susceptibilities and molecular hyperpolarizabilities. For the linear and third order NLO processes of interest here these relations are given by

$$
\chi_{I J}^{(1)}\left(\omega_{1} ; \omega_{2}\right)=f_{1}^{\omega_{1}} f_{j}^{\omega_{2}} \sum_{n} N^{(n)} \sum_{i j} a_{i I}^{(n)} a_{j J}^{(n)} \alpha_{i j}\left(\omega_{1} ; \omega_{2}\right)
$$

and

$$
\begin{aligned}
& \chi_{I J K L}^{(3)}\left(\omega_{4} ; \omega_{1}, \omega_{2}, \omega_{3}\right)=f_{I}^{\omega_{1}} f_{J}^{\omega_{2}} f_{I}^{\omega_{3}} f_{J}^{\omega_{4}} \sum_{n} N^{(n)} \sum_{i j k l} a_{i I}^{(n)} a_{j J}^{(n)} a_{k K}^{(n)} a_{l L}^{(n)} \\
& \quad \times \gamma_{i j k l}\left(\omega_{4} ; \omega_{1}, \omega_{2}, \omega_{3}\right),
\end{aligned}
$$

where $N^{(n)}$ is the number of identical molecules per unit volume in a given subgroup, $f$ 's are local field factors, $a$ 's are direction cosines and $n$ is the number of different subgroups in the unit cell. For a spherical molecule the local field factors at optical frequency are given by the well known Lorentz-Lorenz formula

$$
f^{\omega}=\left(n^{2}+2\right) / 3
$$

where $n$ is the medium refractive index. For a cylindrical molecule $f^{\omega}=1$.

Capital letters in Eqs. (3), (4) refer to the laboratory reference frame, whereas the small ones to the molecular one. Consequently, the macroscopic susceptibility, as it is seen from Eqs. (1), (2) depends not only on the value of molecular polarizability or hyperpolarizability, but also on the orientation of molecules $[2,3]$. In the case of polycrystalline or non-crystalline materials relations (3), (4) are less obvious. However, it is possible to introduce an orientation distribution function $P(\Phi, \Theta, \Psi)$ giving the probability of finding the molecular axis in a given direction described by angles $(\Phi, \Theta, \Psi)$. In that case the linear and the third order NLO susceptibilities are given by

$$
\chi_{I J}^{(1)}\left(\omega_{1} ; \omega_{2}\right)=\sum_{m} \sum_{i j} N^{m} F^{m}\left\langle a_{i I} a_{j J} \mid P(\Phi, \Theta, \Psi)\right\rangle \alpha_{i j}\left(\omega_{1} ; \omega_{2}\right)
$$


and

$$
\begin{aligned}
& \chi_{I J K L}^{(3)}\left(\omega_{4} ; \omega_{1}, \omega_{2}, \omega_{3}\right)=\sum_{m} \sum_{i j k l} N^{m} F^{m}\left\langle a_{i I} a_{j J} a_{k K} a_{l L} \mid P(\Phi, \Theta, \Psi)\right\rangle \\
& \quad \times \gamma_{i j k l}\left(\omega_{4} ; \omega_{1}, \omega_{2}, \omega_{3}\right),
\end{aligned}
$$

where $m$ numbers the species (if different) in a bulk material and $F$ is a global local field factor.

In general there is 9 polarizability $\alpha$ and 81 hyperpolarizability $\gamma$ tensor components. Depending on the molecule symmetry this number can be strongly reduced [4]. Fortunately, the conjugated quasi 1D polymers are characterized by an enhanced linear polarizability $\alpha_{x x}$ and second hyperpolarizability $\gamma_{x x x x}$ tensor components in the polymer chain direction $x$. Consequently, all other tensor components may be neglected. Thus the polymer chain orientation distribution function will depend on one angle $\Theta$ only, which is the angle between the polymer chain direction and a privileged direction in the laboratory frame (e.g. a direction on thin film substrate). The case of mono-orientation (all polymer chains parallel: $\Theta=0$ or $\Theta=\pi$ ) and of bi-orientation (polymer chains parallel to two perpendicular directions, which reduces to the preceding case by considering two, equally populated species with nonzero tensor components $\gamma_{x x x x}$ and $\gamma_{y y y y}$ ) fabricated by epitaxy on monocrystalline substrates have been described and discussed previously $[2,5,6]$. In the present case we will discuss the case of not perfectly oriented thin films with an orientation distribution. As most of applications are targetted with thin films we will limit our discussion here to that case only.

\section{Oriented thin film preparation techniques}

There is an important activity devoted to the fabrication of ordered thin films. For these purposes several methods, applicable to conjugated polymer thin films, have been developed. These are:

i) Langmuir-Blodgett technique,

ii) shear technique,

iii) stretching,

iv) epitaxy.

The first technique requires specially designed amphiphilic molecules with long aliphatic chains and polar groups assuring them a good stability on water subphase. It works with some polydiacetylenes [7] giving polycrystalline thin films with polymer chains parallel to the substrate plane [8]. A recent paper of Tomioka et al. [9] reports a method of preparation of highly oriented Langmuir-Blodgett monolayers using a polydiacetylene with urethane side-groups.

The shear technique has been applied succesfully for very well crystallizing polydiacetylenes like PTS (poly toluen sulphonate) and TCDU (poly [5,7-dodecadiyne-1,12 diol-bis(phenylurethane)]) [10]. The single crystalline thin film growth is obtained from a saturated monomer solution kept between two e.g. silica plates at low temperature. The monomer molecules are preoriented by shear in the melt state or in solution.

The stretching technique applies to free standing films or films deposited on a free standing stretchable thin film substrate [11]. 
The high vacuum evaporation, and consequently the epitaxy technique applies only for topochemically polymerizing molecules like diacetylenes. The main problem here consists in an adequate choice of substrate with crystalline contact plane fitting well with that of deposited molecule. With $\mathrm{KBr}$ single crystal substrate and DCH molecule it was possible to obtain a bi-orientation [6] with polymer chains (after thermal polymerization of deposited monomer thin film) mutually perpendicular and parallel to the cubic edges of KBr. With the choice of KAP single crystal substrate a mono-orientation of polymer chains was obtained [7]. For phthalate acid with other alkali ions like $\mathrm{Rb}$ single crystals the technique leads to an orientation distribution of polymer chains [7].

Kanetake et al. [12] proposed a technique, currently used in oriented thin film preparation from liquid crystals, and consisting in an epitaxy of organic molecule on a preoriented substrate by rubbing.

In the present study thin films were obtained by vacuum epitaxy of diacetylene monomers shown in Fig. 1 . In the case of polymer CNEU (poly $(N$-carbazolyl-5,7-nonadiyne-1ol-1-ethylurethane)) we have used a KAP single crystal substrate. For the second polymer (p-4BCMU, poly (5,7-dodecadiyne-1,12 bis[(4-butoxycarbonyl) methylurethane], cf. Fig. 1) we used the technique applied by Kanetake et

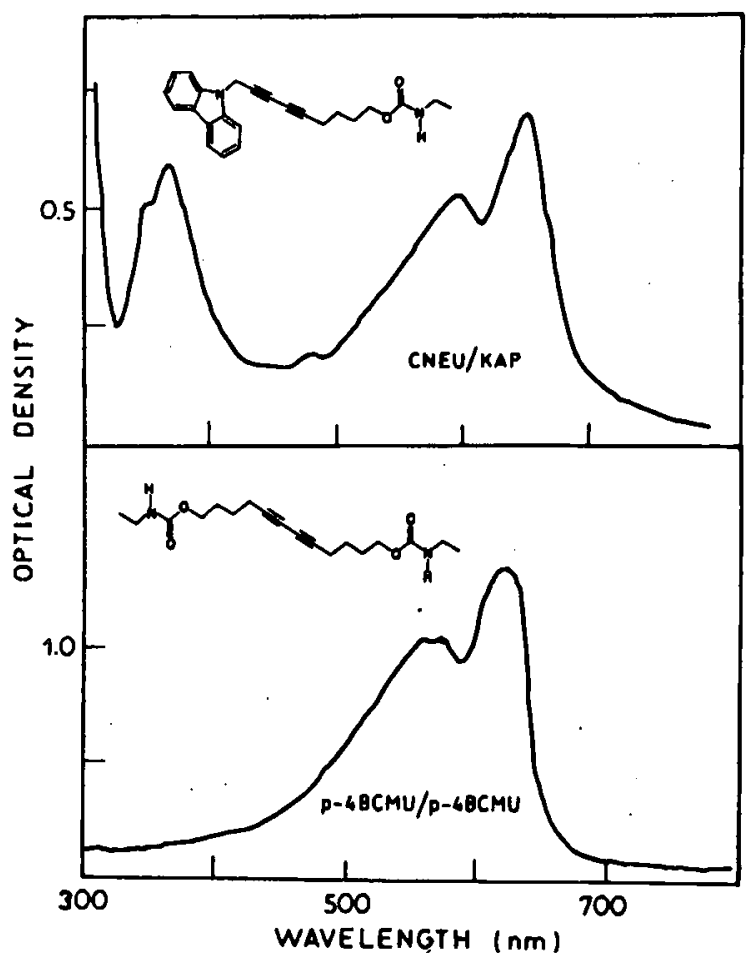

Fig. 1. Monomer chemical structure and optical absorption spectra of studied polydiacetylenes. 
al. [12] for another polydiacetylene. The difference between the two studied polymers consists in the replacement of one 4-BCMU group in p-4BCMU by a DCH group. Consequently the monomer CNEU (cf. Fig. 1) is noncentrosymmetric.

The film of $\mathrm{p}-4 \mathrm{BCMU}$ was obtained in the following way. First we deposited at high vacuum a very thin film (100-150 $\AA$ ) of monomer on a well cleaned silica substrate. After a topochemical polymerization with UV light the polymer chains were oriented by rubbing. Subsequently a thicker film was grown by high vacuum deposition of diacetylene monomer. The epitaxied film was again polymerized with UV light. Up to $3000 \AA$ thickness well oriented thin films can be obtained in this way. The optical absorption spectra of both polymers are shown in Fig. 1. Poly 4-BCMU, which is a soluble polymer, is known for making "red" films from solution casting. In the case of evaporated films one obtains so-called "blue" form of PDA (polydiacetylene), with larger conjugation length due to a better polymer planarity. In both cases no perfect orientation was achieved which was observed by a noncomplete extinction of optical absorption between crossed polarizers.

\section{Orientation distribution function}

As we already mentioned the orientation distribution in the case of conjugated 1D polymers depends often on one angle $\Theta$ only, which is the angle between molecular axis and a privileged direction in the laboratory frame (e.g. a substrate edge). At least we will limit our discussion here to such a situation. In the following we will express the linear and the cubic NLO susceptibilities in terms of order parameters and show how these can be determined using the linear absorption and the third harmonic generation spectra.

In the case of stretched films or for some epitaxied films the chain orientation cannot be described by the Dirac distribution function. The situation is still simple if all chains are parallel to the substrate plane, as it is often the case. In that situation the orientation distribution function depends indeed on one angle $\Theta$ only. In order to get this orientation distribution function one can develop $P(\Theta)$ as a function of $\cos n \Theta$, such a development being simply suggested by the form of cubic susceptibilities (3), (5). We note here that such a development has been recently used by Neher et al. [10] for nonlinear optical dichroism studies of the Langmuir-Blodgett films of poly(bis- $m$-butoxyphenylsilane)

$$
P(\Theta)=\sum_{n} O_{n} \cos n \Theta
$$

Introducing it into Eq. (1) with the use of Eqs. (3), (5) one gets for the linear susceptibility $\chi_{X X}^{(1)}$ tensor component the following expression:

$$
\chi_{X X}^{(1)}=N F \alpha_{x x}\left(2+O_{2} \cos 2 \Theta\right) .
$$

Consequently, the extinction coefficient $\kappa$ linked to $\chi^{(1)}$ through the following relation:

$$
\kappa=\operatorname{Im} \chi^{(1)} / 2 n
$$

where $n$ is the real part of the refractive index, shows a similar angular dependence. By taking the ratio of extinction coefficients for two incident $s$ light polarizations 
corresponding to maximum and minimum absorptions, respectively, one can get a rapid evaluation of the order parameter $\mathrm{O}_{2}$

$$
\frac{\kappa^{\mathrm{MAX}}}{\kappa^{\mathrm{MIN}}}=\frac{2+\mathrm{O}_{2}}{2-\mathrm{O}_{2}} \text {. }
$$

In Eq. (11) we have neglected the refractive index, as well as possible field factors anisotropy. For not perfectly ordered films we expect this anisotropy to be not very important. Its importance will increase with order. The order parameter can be also obtained from the angular dependence of extinction coefficient by fitting Eq. (9) to experimental data as it is shown in Fig. 2 for CNEU polymer.

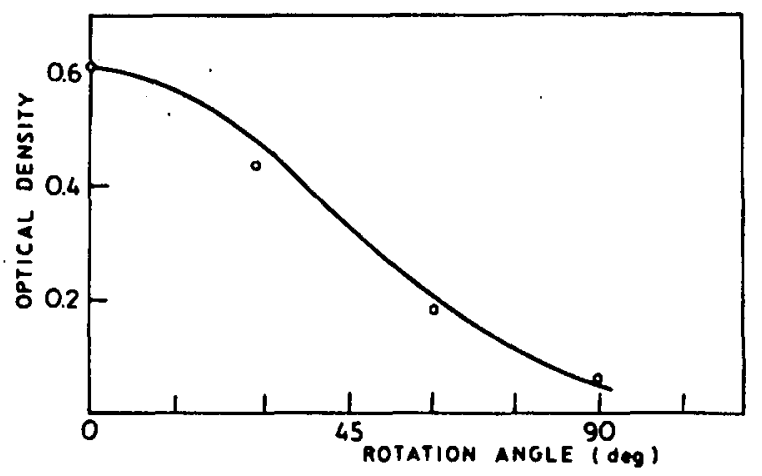

Fig. 2. Rotation angle dependence of optical density measured for a p-CNEU epitaxied thin film. Open circles show measured values whereas solid line - the fitted one, as described in text.

Similarly the diagonal third order NLO susceptibility tensor component $\chi_{X X X X}^{(3)}$, as measured by third harmonic generation (THG), is given by the following expression:

$$
\chi_{X X X X}^{(3)}=N F \gamma_{x x x x}\left(6+4 O_{2} \cos 2 \Theta+O_{4} \cos 4 \Theta\right) .
$$

Consequently, the THG intensity will be proportional to the square of cubic susceptibility and will be given by the following expression:

$$
I_{3 \omega}=A I_{\omega}^{3}\left(6+4 O_{2} \cos 2 \Theta+O_{4} \cos 4 \Theta\right)^{2} .
$$

By rotating thin film along an axis parallel to the beam propagation direction (cf. Fig. 3) one observes a variation of harmonic intensity with the rotation angle. If this one coincides with angle $\Theta$ this variation will be given directly by Eq. (13). The order parameters can be determined by fitting the angular dependence of harmonic intensity in function of rotation angle or simply by coupling THG measurements with linear dichroism data. In fact, the contrast ratio defined by the maxima and minima THG intensities is given by

$$
R=\frac{I_{3 \omega}^{\mathrm{MAX}}}{I_{3 \omega}^{\mathrm{MIN}}}=\frac{\left(6+4 O_{2}+O_{4}\right)^{2}}{\left(6-4 O_{2}+O_{4}\right)^{2}}
$$

which can be used to a fast determination of the order parameter $O_{2}$ if $O_{4}$ is known (from e.g. linear absorption spectra). 


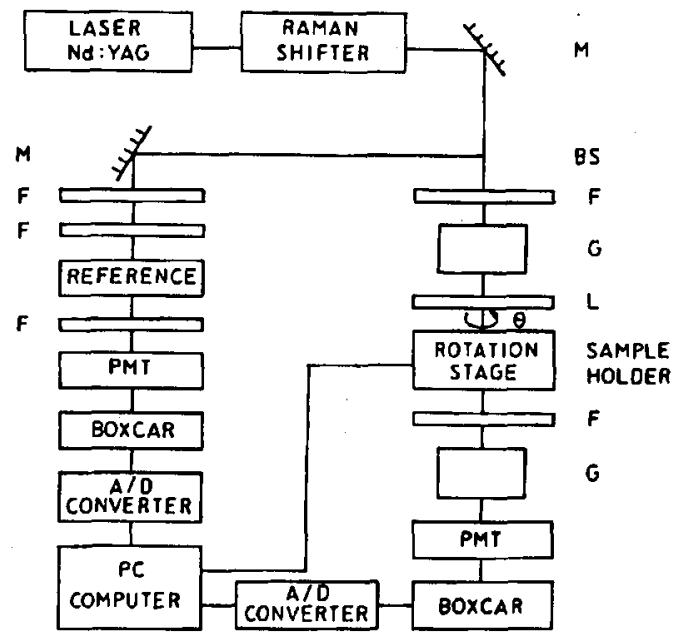

Fig. 3. Schematic representation of experimental arrangement for nonlinear optical dichroism measurements in thin films by third harmonic generation technique. $G-$ polarizer, $F^{\prime}$ s - filters, $L$ - lens, $B S$ - beam splitter, $M$ - mirror. Thin film is rotated along an axis parallel to the beam propagation direction (and perpendicular to its surface) and/or along an axis perpendicular to it.

\section{Results}

Figure 2 shows observed and fitted angular dependence of extinction coefficient for CNEU polymer thin film. Solid line shows the fitted values using Eq. (9). The determined in this way order parameters $\mathrm{O}_{2}$ are listed in Table. The optical absorption spectra were obtained using a polarized incident light and by rotating thin film about an axis parallel to the normal to the thin film substrate.

TABLE

Order parameters $O_{2}$ and $O_{4}$ as determined for the studied polymers.

\begin{tabular}{l|c|c}
\hline \hline Polymer & $\mathrm{O}_{2}$ & $\mathrm{O}_{4}$ \\
\hline $\mathrm{p}$-CNEU & $1.58^{a}$ & 2.12 \\
$\mathrm{p}-\mathrm{BCMU}$ & $0.72^{b}$ & 0.27 \\
\hline${ }^{a}$ measured by linear dichroism, \\
${ }^{b}$ determined from angular variation of THG intensity
\end{tabular}

The $O_{4}$ order parameters were obtained from THG generation measurements on oriented thin films. The used experimental setup is shown in Fig. 4. The light source is an Nd:YAG laser. The measurements were done at $1.907 \mu \mathrm{m}$ fundamental wavelength, obtained by a direct shift of fundamental $1.0642 \mu \mathrm{m}$ by stimulated Raman scattering in a high pressure hydrogen cell. Similarly as in linear optical 

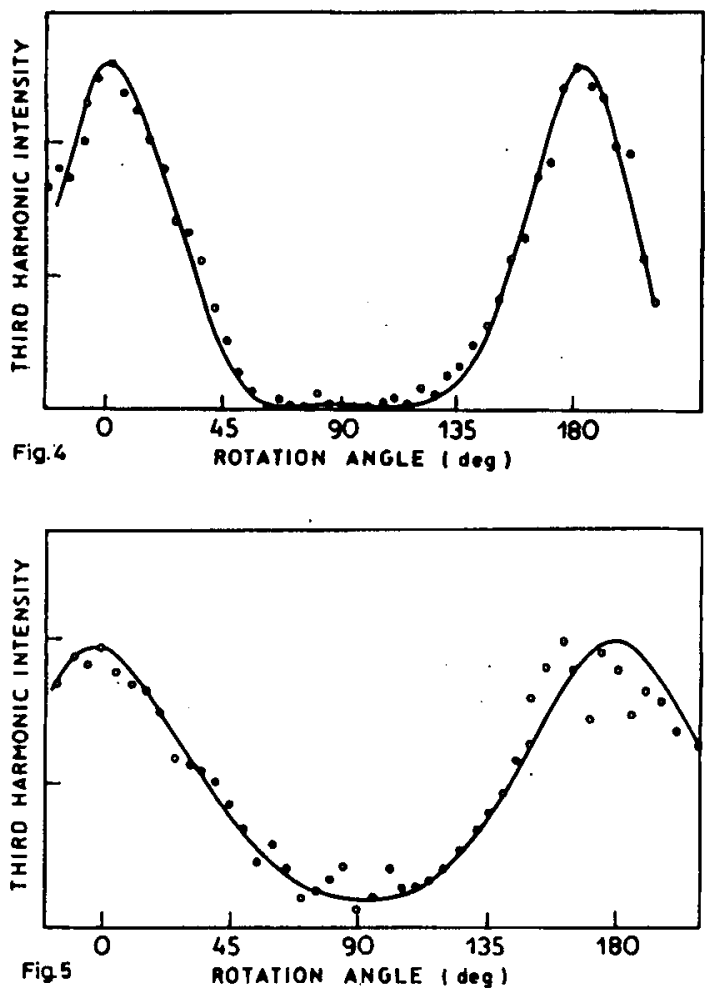

Fig. 4. Rotation angle dependence of third harmonic intensity for a p-CNEU epitaxied thin film on KAP single crystal. Closed circles show measured values whereas solid line - the fitted one, as described in text. Cubic susceptibility dependence on number of double bonds in thiophene oligomers.

Fig. 5 Rotation angle dependence of third harmonic intensity for a p-4BCMU epitaxied thin film on KAP single crystal. Closed circles show measured values whereas solid line - the fitted one, as described in text.

dichroism measurements, the film was rotated along an axis parallel to the beam propagation direction and the harmonic intensity was collected as the function of rotation angle. The rotation angle dependence of harmonic intensity was fitted using Eq. (13). Figures 4 and 5 show the measured and the fitted values of harmonic intensity as a function of the rotation angle for both polymers. An excellent agreement between theory and experiment is seen. In the case of p-CNEU the $\mathrm{O}_{4}$ order parameter was obtained by injecting $\mathrm{O}_{2}$ from the linear absorption spectra variation. For p-4BCMU $\mathrm{O}_{2}$ and $\mathrm{O}_{4}$ order parameters were determined from rotation angle variation of THG intensity. The determined in this way order parameters for both polymers are given in Table.

\section{Discussion}

The nonlinear optical dichroism technique is a powerful tool in the structure study of conjugated in $1 \mathrm{D}$ thin polymer film. The largest thin film thickness used 
is determined by its absorption coefficient. Contrary to the case of linear optical dichroism, where the technique is applicable in the thin film absorption range only, this can be used outside absorption band in the former case. Moreover, the nonlinear optical dichroism technique works also in the case when the linear one fails, as in the case of bi-orientation [6].

In the present case the study was facilitated by the fact that polymer chains are parallel to the substrate plane. It is however possible to introduce orientation distribution function depending on 2 angles $P(\Theta, \Phi)$ in more general cases.

As it is seen from Table the molecular epitaxy on a single crystal substrate gives a higher order, as expected. In the case of p-DCH epitaxy on KAP single crystal an almost perfect mono-orientation of polydiacetylene chains was obtained with $\chi^{(3)} / \chi_{X X X X}^{(3)}>140_{Y Y Y Y}[5]$. This is due to a good matching of the contact planes of epitaxied compound with respect to the substrate. When one dicarbazolyl side group is replaced by a BCMU one (cf. Fig. 1) this matching is not so good and the order is lower. The technique used here and consisting in the preorientation of a very thin layer by rubbing gives still lower order.
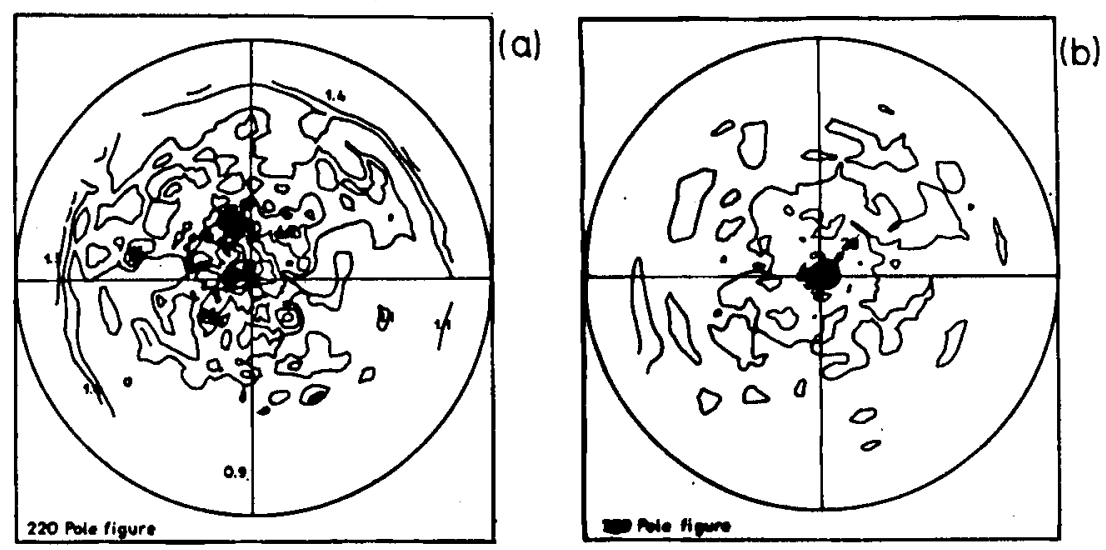

Fig. 6. Pole figures for an isotropic (a) and an epitaxied (b) p-4BCMU thin film on a preoriented layer.

Figure 6 shows pole figures for an isotropic (a) and the oriented (b) p-4BCMU film. If for the isotropic film one observes an almost random distribution of the crystallographic axes, an excellent orientation is observed for p-4BCMU epitaxied film, with a maximum of 28 . It corresponds to a higher order than that obtained by the nonlinear dichroism method (cf. Table). The difference may be due to the fact that in the X-ray study only crystallites are seen whereas the amorphous phases, most likely present in polymer thin film, also contribute to the nonlinear optical response.

We discussed here the easiest case when the polymer chains are parallel to the substrate. In the case of in-plane or in-space randomly distributed polymer chains the average macroscopic cubic susceptibility is given by

$$
\left\langle\chi^{(3)}\right\rangle_{X X X X}=N F \gamma_{x x x x}\left\langle\cos ^{4} \Phi\right\rangle
$$


where $\Phi$ is the angle between polymer chain and the exciting (as well as the resultant) optical electric fields, assumed a priori as having the same direction. In that case one has

$$
\left\langle\cos ^{4} \Phi\right\rangle=\left\{\begin{array}{l}
1 \text { for a mono-orientation (all polymer chains parallel to a } \\
\text { given direction), } \\
3 / 8 \text { for a bi-dimensional disorder (all polymer chains paral- } \\
\text { lel to a plane and randomly distributed within this plane) } \\
1 / 5 \text { for a three-dimensional disorder. }
\end{array}\right.
$$

It shows an increase by a factor of 5 in cubic susceptibility (and a factor of at least 25 in efficiency, depending on third order process under consideration) when passing from a completely disordered system to a mono-oriented one (e.g. single crystal). Again it shows interest of oriented thin films for NLO applications.

\section{References}

[1] See e.g.: Nonlinear Optical Properties of Organic Molecules, Vols. 1-2, Eds. D.S. Chemla, J. Zyss, Academic Press, Orlando 1987; Organic Molecules for Nonlinear Optics and Photonics, Eds. J. Messier, F. Kajzar, P. Prasad, NATO ASI Series, Vol. E194, Kluwer Academic Publisher, Dordrecht 1991.

[2] F. Kajzar, Nonlinear Opt. 5, 329 (1993).

[3] D. Neher, S. Mittler-Neher, M. Cha, G. Stegeman, in: Nonlinear Optical Properties of Organic Materials V, Ed. K.D. Singer, Proc. SPIE, 1560, SPIE, Bellingham, Washington, p. 335.

[4] See e.g. P.N. Butcher, Nonlinear Optical Phenomena, Bulletin 200, Ohio State University, Columbus, Ohio 1965.

[5] J. Le Moigne, F. Kajzar, A. Thierry, Macromolecules 24, 2622 (1991).

[6] J. Le Moigne, A.Thierry, P.A. Chollet, F. Kajzar, J. Messier, J. Chem. Phys. 88, 6647 (1988).

[7] B. Tieke, H.J. Graf, G. Wegner, B. Naegele, H. Ringsdorf, A. Banerjie, D. Day, J.B. Lando, Kolloid Zeit. 255, 521 (1977).

[8] F. Kajzar, L. Rothberg, S. Etemad, P.A. Chollet, D. Grec, A. Boudet, T. Jedju, Opt. Commun. 66, 55 (1988).

[9] Y. Tomioka, S. Imazeki, N. Tanak, Chem. Phys. Lett. 174, 433 (1990).

[10] M. Thakur, S. Meyler, Macromolecules 18, 2341 (1985).

[11] Th. Schimmel, M. Schwoerer, H. Narmann, in: Electronic Properties of Polymers. Orientation and Dimensionality of Conjugated Systems, Eds. H. Kuzmany, M. Mehring, S. Roth, Springer Verlag, Berlin 1992, p. 81; G. Gustafsson, O. Inganas, P. Dyreklew, ibid., p. 329.

[12] T. Kanetake, K. Ishikawa, T. Hasegawa, T. Koda, K. Takeda, M. Hasegawa, K. Kubodera, M. Kobayashi, Appl. Phys. Lett. 54, 2287 (1989). 